\title{
DEEP HST/FOC OBSERVATIONS OF THE CENTER OF M15 ${ }^{1}$
}

\author{
CRAIG SOSIN AND IVAN R. KING \\ Department of Astronomy, University of California, \\ Berkeley, CA 94720-3411, USA
}

\begin{abstract}
We present preliminary results of the analysis of a set of Hubble Space Telescope/Faint Object Camera images of the center of M15, the prototypical post-core-collapse globular cluster. We rule out, at the $95 \%$ confidence level, the $2 ! \cdot 2$ core claimed to be detected in pre-repair HST imaging. We also measure a mass function in a field $20^{\prime \prime}$ from the center.
\end{abstract}

The advent of the repaired Hubble Space Telescope (HST) has made it possible to study the dense cores of globular clusters in unprecedented detail. The telescope's high spatial resolution allows us to observe the distribution of faint stars in these crowded regions-a capability that is crucial is we wish to understand these clusters' dynamical state.

We observed three $7 \times 7^{\prime \prime}$ fields in M15 on 27 September 1994; two near the cluster center, and one at $r \simeq 20^{\prime \prime}$. (A portion of the former images is reproduced in King's paper in this volume.) All were observed in the FOC equivalents of $B(\mathrm{~F} 430 \mathrm{~W})$ and $V$ (F480LP), for $\sim 2000$ seconds in each color. The resulting images show stars down to $V \simeq 22$ in the inner fields, and down to $V \simeq 24\left(\sim 0.5 M_{\odot}\right)$ in the outer field.

We used the standard DAOPHOT software to produce lists of stellar positions and magnitudes, with a few additions - such as an algorithm to reject false detections in diffraction rings - that will be described more fully in an upcoming paper (Sosin \& King, in preparation).

The completeness-corrected surface-density profile of stars with $V$ magnitudes between 18.5 (just above the main-sequence turnoff) and 20.0 is shown in Figure 1. All of these 839 objects have nearly the same mass. The sample is $>90 \%$ complete over most of its radial range. Improvements to

\footnotetext{
${ }^{1}$ Based on observations with the NASA/ESA Hubble Space Telescope, obtained at the Space Telescope Science Institute, which is operated by AURA, Inc., under NASA contract NAS5-26555. This work was supported by NASA grant NAG5-1607.
} 

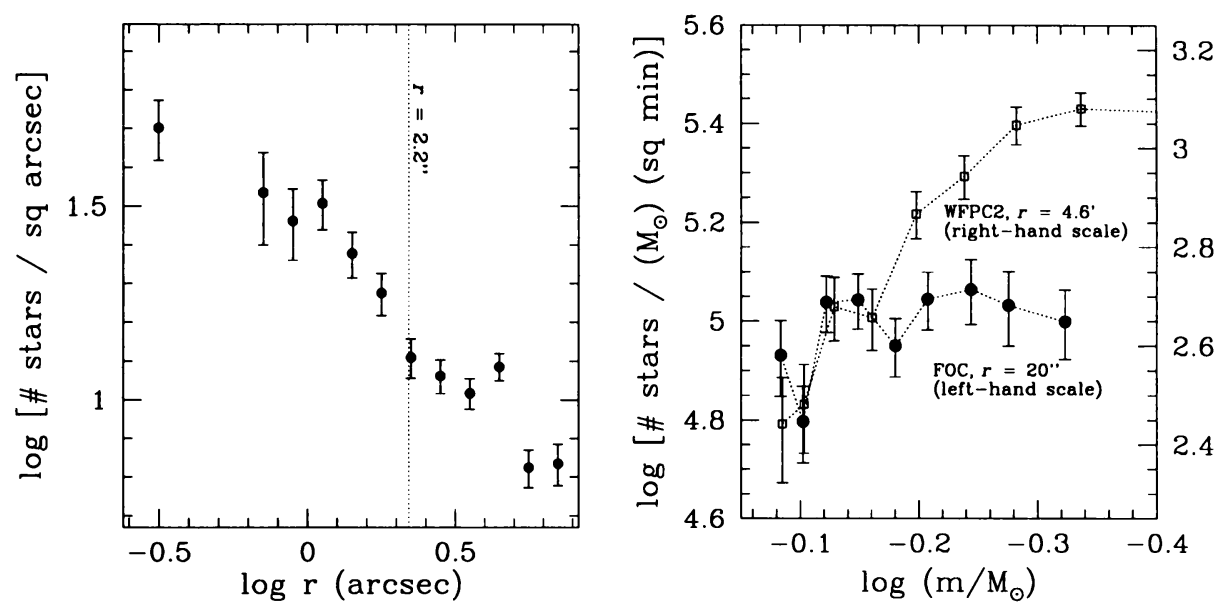

Figure 1. (left) The surface-density profile of stars with $18.5 \leq V \leq 20.0$.

Figure 2. (right) The mass function at $r=20^{\prime \prime}$, and at $r=4 ! 6$.

the bad-object rejection algorithm are currently being developed, and it should soon be possible to extend the analysis to fainter magnitudes.

The surface-density profile clearly continues to climb steadily within $2 "$. The $2 ! 2$ core found in pre-repair HST work by Lauer et al. (1991) (and subsequently questioned by Yanny et al. [1994]) is not seen in these data; using a maximum-likelihood method, we rule out a $2^{\prime \prime}$ core at the $95 \%$ confidence level. We cannot distinguish at present between a pure powerlaw profile and a very small core.

The lesser degree of crowding in the $20^{\prime \prime}$ field allows us to measure a mass function (MF), shown in Figure 2. The counts have been corrected for incompleteness, and the magnitudes converted to masses via the mass-luminosity relation of D'Antona \& Mazzitelli (1995). The best-fitting power-law slope of the MF at $r=20^{\prime \prime}$ is $-0.99 \pm 0.5$, where the Salpeter value is 1.35 .

We also show the mass function at $r=4 ! 6$, as measured in a WFPC2 image (Piotto, Cool, \& King, in preparation). The zero points of the two plots (i.e., the left- and right-hand scales) are chosen so that the two MFs appear to overlap at the high-mass end, to emphasize their substantial difference at lower masses-the result of dynamical mass segregation.

\section{References}

D'Antona, F. \& Mazzitelli, I. 1995, preprint, "Stellar models and luminosity functions for the Population II main sequence down to its low end"

Lauer, T. et al. 1991, ApJ, 369, L45

Yanny, B., Guhathakurta, P., Bahcall, J., \& Schneider, D. 1994, AJ, 107, 1745 